\title{
Rechtsanwalt Hans Heinz Heldmann: Plädoyer zum Stammheimer Prozeß
}

Eine erste Nachrede auf das Justizverfabren von Stammbeim, das fortgesetzter juristischer Auszebrung erlegen ist*

Dieses Verfahren, von seinen Anfängen an rechesbrüchig, wovon hier zu sprechen sein wird, ist von denen, die es betrieben haben, zu seinem eigenen Phantom malträriert worden. Den Fangschuß haben ihm die kraft Amtes berufenen Hüter von Justiz und Verfassung gegeben. Danach war für die Verteidiger der Stammheimer Gefangenen innerhalb der Justizfestung nichts mehr zu sagen. Darum sprechen wir heute und in den nächsten Tagen zu einer Offentlichkeit außerhalb des Kriegsschauplatzes Stammheim.

Am 12. April 1977 habe ich dem Gericht zwei Einstellungsanträge vorgelegt. Sie sagen:

der erste: der Beschluß des Bundesgerichtshofs (BGH), ohne die Angeklagten die Hauptverhandlung fortzusetzen, ist null und nichtig; denn er beruht ursächlich

a) auf einer Verkehrung der ihm zugrundeliegenden medizinischen Gutachten der gerichtlich bestellten Sachverständigen

b) auf der maßgeblichen Mitwirkung eines ungesetzlichen, nämlich offenkundig befangenen Richters, welche das Gesetz verbietet;

der zweite: mit dem Mitte März 1977 aufgedeckten Abhören von Verteidigergesprächen seit zwei Jahren sind die verfassungsrechtlich wie gesetzlich garantierten Grundlagen der Strafverteidigung zerstört worden, und zwar irreparabel,

a) als illegaler Eingriff der Exekutive in das justizielle Verfahren, welches als Rechtsstaatsgebot ein sfaires Verfahren klagten in seinem Verhältnis zum Ankläger garantiert,

b) als die Vernichtung des Schweigerechts des Angeklagten, welches die absolute Vertraulichkeit seines Verteidigergesprächs einschließt.

Für die Tatbestände wie für ihre rechtliche Würdigung verweise ich auf den Text meiner beiden Anträge vom 7.2. und 9.4. 1977, welche dem Manuskript als Anlagen $I$ und 2 angeheftet sind. [...]

Ebenfalls am I 2. April 1977 hat die Verteidigung den Richter Dr. Berroth wegen Besorgnis der Befangenheit abgelehnt.

Richter Berroth nämlich hatte am Gründonnerstag, 7.4., über die Gefangenen, die sich im Hungerstreik gegen die von den medizinischen Sachverständigen erst neuestens wieder eindringlich verurteilten Haftbedingungen befanden und befinden, die Totalisolation verhängt: Einzeleinsperrung, Verbot von Rundfunk und Fernsehen, Verbot jeglichen Besuchs und jeglicher Kontaktaufnahme zu ihren Verteidigern - der nächste Bruch desselben Rechts, hier: der faktische Verteidigerausschluß. Nach seiner eigenen Erklärung hat Richter Berroth das auf bloßen Telefonanruf eines Bundesanwalts hin verfügt.

Der war um I I.25 Uhr, seine Anordnung erging um I I.30 Uhr - s Minuten später. Begründet hat er sie nicht - er hat "Sicherheitsbelange* deklariert; "Sicherheitsbelangek also stünden dem gesetzlich privilegierten Verkehr von Angeklagten und Verteidigern entgegen. Auf seine Ablehnung am 12. 4. 1977 hat Richter Berroth

\footnotetext{
* Dokumentation der Rede einer der Wahlverteidiger, gehalten in einem Stuttgarter Hotel am 27. April 1977, dem Tag vor der Urteilsverkündung.
} 
seinen Verteidigerausschluß vom 7. 4. 1977 zu erklären versucht: „Mir war dabei bekannt, daß nach der Anstaltsordnung ohnehin keine Anwaltsbesuche über Wochenenden und an Feiertagen möglich sind.«

Was - will der Richter selber hier die Nichtsnutzigkeit seines Richterhandelns bekennen?

Am 9. 4. 1977 allerdings hatte er sich noch nicht soweit besonnen, sondern gegenüber Rechtsanwalt Müller erklärt: ,Für Ausnahmefälle ist eben diese Verfügung gedacht, daß eben jetzt in den nächsten Tagen kein Anwaltsbesuch (auch nicht mit Ausnahmegenehmigung) stattfinder.،

Uber meine Einstellungsanträge ist das Gericht stillschweigend zu seiner Tagesordnung übergegangen: am nächsten Sitzungstag hat es Beweisaufnahme fortgesetzt - während Erkennen und Rüge eines Prozeßhindernisses jedes weitere Prozedieren ausschließen.

Und den Richter Berrorh, der, ohne Gehör zu gewähren und ohne zu begründen, Verteidigerausschluß anordnet, weil er blindlings wie rechtsblind Verteidiger einer Gefährdung der Sicherheit verdächtigt, haben seine Richterkollegen vom Verdacht der Befangenheit freigesprochen. Was hätte zum Abschluß dieses Verfahrens dessen spezifischen Charakter noch einmal deutlicher machen können? Da schließt der unabhängige Richter auf Telefonanruf der Bundesanwaltschaft ohne zu fragen die Verteidiger aus. Jeder Amtsinspektor prüft vor Eingriff die Rechtslage. Unser Oberrichter prüft gar nichts - er pariert, wenn die Bundesanwaltschaft klingelt; und versucht hernach, aus seinem Rechtsbruch sich herauszureden.

Das gab von Anfang an diesem Verfahren seine Einmaligkeit: daß Richter ihre Vorverurteilungen der Angeklagten offen wie eine Standarte vor sich hergetragen haben - um sogleich, auf prozessuale Beanstandung, deren Offenbarung völlig unangerührt zu leugnen. So endet die Geschichte dieser Hauptverhandlung, wie sie Jahre vor deren Beginn angefangen hat. Und so werden voraussichtlich morgen wir es hören: das Vorurteil als Endurteil.

\section{Der I. Rechtsbruch: Vorverurteilung als innerstaatliche Feinderklärung}

Auf die bis zum Beginn der Hauptverhandlung bereits verfestigte öffentliche Vorverurteilung durch Politiker, Justiz und einen Teil der Presse haben wir von Anfang dieser Hauptverhandlung an innerhalb (z. B. Antrag Schily vom 20. 8. 1975, Tonbandniederschrift [TN] 2244) wie außerhalb (z. B. KONKRET, Heft 8/1975) des Prozesses hingewiesen.

Erinnern Sie wenige Beispiele:

Wie schon im Februar 1971 der damalige Verfassungsminister Genscher der großen Gemeinde der "Bild «-Leser die Unschuldsvermutung unseres Rechtsstaatsprinzips interpretiert hat: ,unter Hinweis auf die Gewalttaten der Baader-Mahler-Bande jeden davor warnt, diese Verbrecher in irgendeiner Weise zu unterstützen، (nach Presseberichten vom I 5. 2. I97I);

wie kurz vor Prozeßbeginn, am 25. 4. 1975 , der Bundeskanzler vor dem Bundestag dem Stammheimer Gericht schwierige Rechtsfindung abgenommen hat: \#... der harte Kern der kriminellen Baader-Meinhof-Vereinigung, dem zahlreiche Morde, Mordversuche, Sprengstoffattentate, Banküberfälle zur Last zu legen sind ... die Gewaltverbrecher Baader, Meinhof und andere ... anarchistische Banditen ...* - und ihm gleich den rechten Weg wies: „... die Ausschöpfung der von der Strafprozeßordnung eröffneten neuen Möglichkeiten ... bis an die Grenze dessen zu gehen, was vom Rechtsstaat erlaubt und geboten ist.«; 
wie im gleichen Stil souveräner Rechtsverachtung auch Kanzlerkandidat Kohl sich zu qualifizieren suchte, indem er lange vor Schluß der Stammheimer Beweisaufnahme deren Ergebnis vorprogrammierte: es »könne eine Begnadigung dieser Mörder und Schwerstverbrecher nicht ins Auge gefaßt werden « (Welt« vom 2. 7. 1976). Drei hervorragende Repräsentanten des freiheitlichen Rechtsstaats, beispielgebend für Aufbereitung öffentlicher Meinung durch Eingriffe in justizielle Verfahren, welche in Gesellschaften, die Demokratie und Bürgerrechte sich erstritten haben, statt als Import sie zu empfangen, schwerer Verletzung richterlicher Unabhängigkeit wegen geahndet werden würden (vgl. z. B. Max Grünhut in Monatsschrift für deutsches Recht 1948, 442 ff., und in Zeitschrift für die gesamte Strafrechtswissenschaft 1962, $319 \mathrm{ff} ., 332 \mathrm{f}$.$) , in den Fällen dieser Beispiele zumindest mittlere$ Regierungskrisen bewirkt hätten.

Richard Harvey, Mitglied der English Bar, Prozeßbeobachter in Stammheim, hat Rechtsstaats-Standard gekennzeichnet, wo er die öffentliche Äußerung des höchsten Anklägers: Baader habe sein Recht auf einen fairen Prozeß verwirkt ("Stern " vom 5. 6. 1975), nach angelsächsischem Maßstab als absoluten Grund für sofortige Amtsentfernung gewürdigt hat.

Bis zum Prozeßbeginn hatte längst juristische wie nicht-juristische Offentlichkeit an den Stil beschimpfender Vorverurteilung sich gewöhnt. Daß die Sitzungsvertreter der Bundesanwaltschaft vom Beginn der Hauptverhandlung an hemmungslos von den "Bandenangehörigen ", vom "Rädelsführer Baader ", von den "folgenschwersten Verbrechen der Baader-Meinhof-Bande* (vgl. TN vom 20.6.1975, 712-717) in öffentlicher Hauptverhandlung gesprochen haben, hat gar keinen Anstoß erregt. Die Enthemmung der Justiz war schon zu jener Zeit des Prozeßbeginns soweit fortgeschritten, daß die Richter Foth, Mayer und Berroth solche Exzesse der Bundesanwaltschaft wortwörtlich in ihren Beschluß vom 20. Juni I975 aufgenommen haben, ohne eine distanzierende oder auch nur kritische Anmerkung dazu zu finden; dazu auch noch die Publizierung der unglaublichen Auslassungen dieser Bundesanwälte nicht scheuten, die Verteidigung propagiere die physische und psychische Vernichtung von Richtern (TN 715,717 ).

Als die Verteidigung dem heute präsidierenden Richter Foth drauf kam (TN vom 10. 1. 1977, 13165-13167), daß er schon fast drei Jahre vor Anklageerhebung in diesem Verfahren, am 2. I1. 1971, in richterlicher Entscheidung von $* B a a d e r-M e i n-$ hof-Bande « gesprochen hatte (OLG Stuttgart, 3 Ws 288/71), da bewirkte der Vorwurf richterlicher Voreingenommenheit nichts anderes als die Aufklärung, der Ausdruck »Bande* sei »eine weit verbreitete schlagwortartige Bezeichnung , frei von Wertung. (Weit verbreitet freilich war der Begriff schon rund 30 Jahre früher durch deutsche Bandenbekämpfung in Rußland.)

\section{Der nächste Rechtsbruch: die Gesetzesänderungen}

Stimuliert von öffentlicher Urteilsbildung außerhalb der Gewaltentrennung hat zwischen Anklageerhebung und Hauptverhandlung der Gesetzgeber die roojährige Strafprozeßordnung speziell auf die Bandenprozesse umgeschneidert.

Damit hat er gegenüber der Stammheimer Blockanklage ohne Individualisierung von Tatbeiträgen die dieser korrespondierende Blockverteidigung zerschlagen; hat über Jahre hinweg eingearbeitete Verteidiger zum Ausschluß entsprechend dem Befinden ihres Prozeßgegners, der Bundesanwaltschaft freigegeben; hat das im politischen Prozeß notwendige Erklärungsrecht der Verteidigung aufgehoben und damit die Prozeßsubjektstellung des Angeklagten reduziert; hat Hauptverhandlung 
ohne Angeklagte zugelassen, was dank findiger Hilfe durch den BGH zu dem makaber-genialen Patent-Rezept führte:

man sperre Bandenmitglieder erst einmal lange genug in Isolation, wegen der "Sicherheitsbelangen natürlich, um ihre alsdann eingetretene Skelettierung auf ihre besondere Gefährlichkeit zurückzuführen, sie demnach als selbstverschuldet verhandlungsunfähig aus ihrer Hauptverhandlung entfernen zu können.

Für die Begründung dieses Abbaus von Verteidigungsrechten stehen scheinbar einträchtig fromme Beschwörungsformeln und militante Polemik nebeneinander:

*Die erste Beratung dieses Entwurfs eines Zweiten Gesetzes zur Reform des Strafverfahrensrechts im Bundestag brachte wiederholte Bekenntnisse zum , Grundsatz der freien Advokatur als auch dem Recht des Beschuldigten auf umfassende Verteidigung، (Bundesminister Dr. Vogel auf S. 8229), zur ,Ordnung des Strafprozessesı als >Magna Charta des Rechtsstaats، Denn der fundamentale Unterschied zwischen einem Rechtsstaat und einem Machtstaat offenbart sich nicht zuletzt darin, wie ein Staat mit einem Beschuldigten, mit einem angeklagten Bürger umgeht, wie er die Rechte dessen ausgestaltet, demgegenüber er von seiner Strafbefugnis Gebrauch macht, (Beifall) ...* (Bundesminister Dr. Vogel auf S. 8230), zum *Recht des Beschuldigten, sich des Verteidigers seines Vertrauens zu bedienen. Die freie Advokatur in ihrer historischen Entwicklung war immer eines der wesentlichen Kennzeichen unseres Rechtsstaates. Wer in autoritären Systemen beobachtet, daß die freie Advokatur zunächst immer Einschränkungen und Gängelungen und schließlich Unterdrückungen ausgesetzt ist, der wird bis zum Außersten mit Generalklauseln oder generalklauselartigen Formulierungen in diesem Bereich zurückhaltend sein.* (Abg. Engelhard, FDP, auf S. 8234)

Aus der dritten Beratung des Gesetzentwurfs im Bundestag (138. Sitzung am I8. I2. 1974)

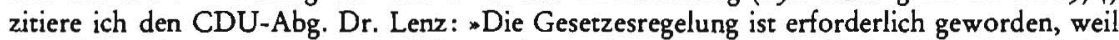
eine kleine Gruppe von etwa zwei Dutzend Anwälten ganz bewußt die Streichung der früher gegebenen Úberwachungsmöglichkeiten dazu mißbrauchen, eine revolutionäre Tärigkeit zu unterstützen. Wer so handeit, der ist kein Organ der Rechtspflege mehr. ... Nur um die Bekämpfung dieser Anwälte handelt es sich hier. Hier handelt es sich nicht darum, die Möglichkeiten der normalen Strafverteidigung einzuschränken. Denn eine normale Strafverteidigung haben diese Verteidiger, diese sogenannten Verteidiger, muß ich ja sagen, überhaupt nicht im Sinn. Sie betreiben ihren revolutionären Kampf im Zusammenspiel mit ihren Mandaten. ...* (S. 9515 des Sitzungsprotokolles)

Monate später beschloß der Gesetzgeber die Uberwachung der Verteidigerpost, so daß seit September 1976 eine schriftliche Verbindung zu unseren Mandanten nicht mehr stattfindet. Die vom Gesetzgeber verworfene Uberwachung des Verteidigergesprächs hatte unheimlich-heimlich die Verwaltung in die Hauptverhandlung bereits eingeführt. Wenn ich diese lex RAF als Rechtsbruch würdige, so übersehe ich nicht, daß einen Teil dieser Neuschöpfungen ( $\$ \int 138 \mathrm{a}, 146,23$ ra StPO) das Bundesverfassungsgericht abgesegnet hat: sie seien verfassungskonform.

Aber auch danach blieb die Frage offen, ob es rechtens ist, daß der Gesetzgeber im Dezember 1974 - zwischen Anklageerhebung im September 1974 und Beginn der Hauptverhandlung im Mai 1975 - in dieses bereits anhängige Verfahren hinein und erklärtermaßen allein zu diesem $Z$ weck das Recht ändern durfte. Erinnern Sie sich bitre, daß Baader zu Beginn der Hauptverhandlung keinen Verteidiger seines Vertrauens mehr hatte; daß die über Jahre erarbeitete Konzeption der Verteidigung, welche nach zahlreichen Zellenrazzien die Behörden längst in Händen hatten, durch Gesetzesänderung wie die Besetzung der Verteidigerbank selbst zerschlagen war.

Das illustriert u. a. Waffengleichheit«: Einem auf dieses Verfahren seit Jahren spezialisierten Anklägerteam stand nur ein Torso noch von Verteidigung gegenüber; und just der "Rädelsführer « hatte gar keinen. Keiner der anderen Verteidiger durfte mit ihm sprechen, keiner durfte für ihn sprechen.

Die Rechtswidrigkeit dieser Gesetzesänderungen vom Dezember 1974 liegt also in der totalen Aufhebung des Verfassungsprinzips der *verfahrensrechtlichen , Waffengleichheit von Ankläger und Beschuldigten* (BVerfGE ${ }_{38} 8$, I I I). 
Den Sondergesetzen korrespondierte das ungesetzlich konstituierte Gericht, was Herr Schily bereits am 2. Verhandlungstag, 5. 6. 1975, als Prozeßhindernis gerügt hat (TN I 23-I 30) (vgl. Schily-Antrag in Anlage 6); erfolglos, weil ein ungesetzlicher Richter kein Prozeßhindernis sei (TN I $_{4}$ ) . Natürlich ist das Gegenteil richtig, wie schon Art. I0I I GG ausweist. Am zweiten Verhandlungstag immerhin noch mußte diese Rechtsfindung überraschen: ein verfassungswidriges Prozedieren hindere dessen Fortführung nicht.

IV. Die Perpetuierung von Rechtsbrüchigkeit (I): das parteiliche Gericht - der befangene Richter

Wie jeder Prozeßbeobachter weiß, hat der Senat in ständiger Rechtsprechung die Vorschriften der StPO über die Richterablehnung als in diesem Verfahren unanwendbar behandelt. Da langte nicht einmal der Nachweis einer Konspiration zwischen dem befangenen Gerichtsvorsitzenden Prinzing und dem befangenen stellvertretenden Vorsitzenden des Revisions- und Beschwerdesenats Meyer; nicht einmal die Beurkundung, wie dieser gegenüber jenem einen Verteidiger einer Unterstützungshandlung verdächtigt hat. Erst als daraufhin die Verteidigung ihre unmittelbare Verfassungsbeschwerde gegen die ungefähr 80. unbegründete Zurückweisung ihrer Richterablehnungen öffentlich angekündigt hatte, setzte der Senat der öffentlichen Peinlichkeit ein Ende.

Danach jedoch ist dieses Rechtsdefizit dieses Verfahrens nicht behoben: daß befangene Richter ungesetzliche Richter sind. So findet dieses Gericht nichts dabei, daß einer der ihren mal eben ein Gesetz außer Kraft setzt, weil sich das zu Ostern so günstig einrichten läßt.

Hat Richter Prinzing mit seiner Direktverbindung zum Revisionsrichter Meyer nichts gewußt von den illegalen Ohren in den Zellen? Hielt er nicht auch sehr flink-intensive Zwiegespräche mit Herrn Nusser (Anstaltsleiter) im benachbarten Einzweck-Gebäude?

Woher wußte Richter Prinzing »konkret«, daß Frau Enßlin ein bestimmter Antrag negal a sei (Stellungnahme vom 20. I. I977 des Rechtsanwalts Künzel)? Von wem hat Richter Prinzing als "Tatsache" erfahren, Baader habe im Spätsommer I975 wörtlich erklärt: "Wir müssen krank sein. Wir müssen gebrochen wirken." (TN 31 39)? Warum weigert beharrlich sich der Senat, die Frage zu beantworten, ob nicht am I 3 3. I975 - zwei Tage nach dem Abhörbeschluß der Sturtgarter Minister - der an jenem Tag unerreichbar gebliebene Richter Prinzing zu entsprechender Instruktion sich im Ministerium befunden hat?!

V. Die Perpetuierung von Rechtsbrüchigkeit (2): Verhandlung trotz Verbandlungsunfähigkeit - Verbandlung obne Angeklagte

I. Seiner bis zum letzten Verhandlungstag fortdauernden Aktualität wegen zitiere ich aus dem gemeinsamen Schriftsatz vom 20. 8. 1975 der gewählten Verteidiger, die am Vortag aus Protest gegen unbeschränktes Weiterverhandeln gegen offensichtlich verhandlungsunfähige Angeklagte den Sitzungssaal verlassen hatten:

»Der gestrige Antrag der Bundesanwaltschaft, uns zu entpflichten, ist zurückzuweisen. Unsere Erklärung, vor abschließender Begutachtung der Angeklagten über ihre Verhandlungs- 
fähigkeit nicht weiter zu verhandeln, ist berechtigt und begründet: Der Verteidiger ist in erster Linie berufen, über die Gesetzlichkeit des Verfahrens zu wachen. Er ist, auch als Pflichrverteidiger, selbständiges Organ der Rechtspflege und steht in voller Gleichberechtigung Gericht und Staatsanwaltschaft gegenüber ...

Prozeßrecht und Standesrecht verweisen den Verteidiger auf Handeln in eigener Verantwortung, als Pflichrverteidiger insbesondere auch darauf, eigene Interessen - etwa: seine Bestellung für einen mittellosen Angeklagten zu erhalten - denen des Mandanten an unbedingtem Einhalten seiner Schutzpflichten unterzuordnen.

Der vom Gericht bestellte Pflichtverteidiger hat ganz besonders darauf zu achten, seine Unabhängigkeit vom Gericht zu wahren - auch gegen die Androhung, durch sein prozessuales Handeln seine Bestellung zu riskieren. Verhandlungsfähigkeit ist Prozeßvoraussetzung. Fehlt sie, ist das Verfahren einzustellen. Sie ist als Verfahrensvoraussetzung in jeder Lage des Verfahrens von Amts wegen zu beachten.

Die Verhandlungsfähigkeit seines Mandanten $m u \beta$ der Verteidiger - begründen sich Zweifel - zum Gegenstand seines Prozeßvortrags machen: ... Die Verhandlungsunfähigkeit steht der Abwesenheit gleich. Sie ist nachträglich allerdings schwer zu beweisen, besonders wenn sie nicht geltend gemacht worden ist und das Gericht sie nicht selbst festgestellt hat. Die Verteidiger scheitern auch hier an der Verwirklichung der Rüge, wenn sie es verpaßt haben, eine Unterbrechung zu beantragen und einen Gerichtsbeschluß herbeizuführen, notfalls den Sitzungssaal zu verlassen.

Die Verteidigung hat seit Beginn der Hauptverhandlung den Mangel der Verhandlungsfähigkeit der Angeklagten stets erneut gerügt. Auf Betreiben der Verteidigung wurde die Frage der Verhandlungsfähigkeit unmittelbar oder mittelbar an folgenden Sitzungstagen zum Gegenstand der Hauptverhandlung gemacht: I1. 6., 12. 6., I8. 6., 1. 7., 2. 7., 8. 7., 9. 7., 10. 7., I6. 7., 24. 7., 30. 7., 31. 7., 5. 8., 7.8. 1975. An vierzehn von sechsundzwanzig Sitzungstagen war somit die Frage der Verhandlungsfähigkeit der Angeklagten Gegenstand der Erörterung in der Hauptverhandlung. ... Seit dem 8. 7. 1975 sehen sich die Verteidiger der Tatsache konfrontiert, daß der Senat selbst die Bestellung von Gutachtern zur Beurteilung der Verhandlungsfähigkeit der Angeklagten für notwendig hält, andererseits jedoch stets dann, wenn der geschwächte gesundheitliche Zustand der Angeklagten Anlaß gab, den Abbruch einer Sitzung oder deren Unterbrechung zu beantragen, lakonisch darauf verwies, die Angeklagten seien nach seiner Auffassung verhandlungsfähig. Diese Auffassung vertritt der Senat bislang, ohne sich auf irgendeine definitive gutachterliche Äußerung der von ihm selbst berufenen Sachverständigen stützen zu können. Den Verteidigern ist in der Rechtsgeschichte der BRD kein Fall bekannt, in dem ein Gericht über annähernd sechs Wochen an der Fiktion der Verhandlungsfähigkeit von Angeklagten festhält, ohne sich auf endgültige Gutachten der von ihm selbst berufenen Sachverständigen stützen zu können.

Am gestrigen Sitzungstag waren die von den beiden Sachverständigen provisorisch dem Senat mitgeteilten weiteren drei Wochen Verhandlungsfähigkeit der Angeklagten abgelaufen. Spätestens zu diesem Zeitpunkt war es mit Verteidigerpflichten nicht mehr vereinbar, sich an der Aufrechterhaltung der vom Senat praktizierten Fiktion der Verhandlungsfähigkeit der Angeklagten zu beteiligen. ... Der Senat hat uns gestern zum Vorwurf gemacht, daß wir unsere Pflichten als Pflichtverteidiger bbeharrliche vernachlässigten. Demgegenüber ist allerdings unsere Verpflichtung, beharrlich darauf zu insistieren, daß die Frage der Verhandlungsfähigkeit der Angeklagten nicht weiterhin zum Spielball richterlicher Willkür gemacht wird.*

Am selben Tag, am 20. 8. 1975, haben die beiden vom Gericht am 21.7. 1975 bestellten Sachverständigen diesem geschrieben: „. . . daß alle vier Angeklagten bis auf weiteres zwar verhandlungsfähig sind, aber nicht in der Lage sein dürften, mehr als drei Stunden pro Tag die Verteidigung in verständiger und verständlicher Weise zu führen und Prozeßerklärungen abzugeben oder entgegenzunehmen. $\propto$

Wer hatte am 19. 8. 1975 - und schon seit Monaten - seine "Pflicht ... gröblich verletzt«?!

2. Als, wiederum einen Monat später, die vom Gericht bestellten Sachverständigen zu endgültigem Ergebnis gekommen sind: einer auf rund drei Stunden täglich beschränkten Verhandlungsfähigkeit - hat das Gericht am 30. 9. I975 beschlossen: "Die Hauptverhandlung wird in Abwesenheit der Angeklagten fortgesetzt."

Um zu diesem Ergebnis zu kommen, hat der Senat rechtliches Gehör für Angeklagte und Verteidiger vereitelt; 
die medizinischen Gutachten der von ihm selbst bestellten Sachverständigen in der entscheidenden Frage - Ursache für mangelnde Verhandlungsfähigkeit - geradezu auf den Kopf gestellt;

seinen sechzehn Seiten langen Beschluß mit einer Fülle von Tatsachenbehauptungen begründet: unbewiesenen - ungeprüft aus Angaben der Bundesanwaltschaft übernommenen - durch Zitateverkürzungen irreführenden - oder einfach wahrheitswidrigen. (Vgl. Anlage 7: Beschwerdeschrift vom 6. 10. 1975).

3. Die Beschwerdeentscheidung vom 22. 10. 1975 des BGH, maßgeblich zusammengebastelt durch den ungesetzlichen Bundesrichter Meyer, verkündet unverhüllt den Ausnahmezustand: die Aufhebung der Menschenrechte auf Leben und körperliche Unversehrtheit.

\section{Die Perpetuierung von Rechtsbrüchigkeit (3): Zerstörung der Verteidigung}

I. Dem Ausschluß der drei neben Rechtsanwalt Schily am längsten eingearbeiteten Verteidiger (Croissant, Groenewold, Stroebele) folgten Entpflichtungen und Zurückweisungen von weiteren zehn Verteidigern. Der Torso von Verteidigung war zerschlagen.

2. Die Bundesanwaltschaft hat gegen alle in Stammheim aufgetretenen Verteidiger der Angeklagten Strafverfahren und/oder Ehrengerichtsverfahren angezettelt: wegen ihrer Verteidigung in diesem Verfahren - noch wäbrend des Andauerns dieser Verteidigung;

eine in der Geschichte zivilisierter Staaten einmalige Praxis! Selbst in dem von der Terrororganisation SAVAK beherrschten Freundesstaat Iran beginnt die Verfolgung von Verteidigern, die sich als solche vor den dortigen Militärtribunalen ernstnehmen, erst nach Abschluß ihrer Verteidigertätigkeit.

Nur einer gewissen Aktualisierung Ihnen bekannter Illustrationen wegen: Schily und ich haben erst vor zwei Wochen neue Strafverfolgung empfangen - wegen eines Beweisantrags in der Hauptverhandlung.

Das Unmaß von Verteidigerverfolgung wird Ihnen unglaublich erscheinen, der Inhalt der Anschuldigungen, für deren fortgesetzte Herstellung die Bundesanwaltschaft emsig sorgt, vielfach als schiere Torheit. Lassen Sie mich jedoch insoweit, bitte, verweisen auf drei Neuerscheinungen aus den letzten Wochen:

Rühmann, Anwaltsverfolgung in der Bundesrepublik, Hamburg, April 1977;

Die Verteidigung auf der Anklagebank (Dokumentation), Hamburg, März 1977;

Ausschaltung politischer Verteidigung (Dokumentation), Frankfurt, Februar 1977.

Die Terrorisierung (lateinisch: terrere = ängstigen, abschrecken) der Verteidiger durch ihre Prozeßgegner, Bundesanwälte und Staatsanwälte, ist bestimmt und geeignet, Verteidigung von "Staatsfeinden a zu unterbinden.

Verteidigerauswahl in diesen Verfahren wird damit ganz zur Sache der Justiz werden. Und da vollzieht sich - gleichsam subkutan - bemerkenswerte Rechtsentwicklung.

In einem vielbeachteten Reformvorschlag "Neuordnung des Strafverfahrens* lesen wir: "Der Vorsitzende muß in die Lage versetzt werden, den Verteidiger auszuschließen. Dem Ausschluß hat eine Vertagung und eine Mitteilung an die Standesorganisation zu folgen ... die Stellung des Verteidigers (ist) . . . zur staatlich gebundenen öffentlich-rechtlichen Tätigkeit geworden . . . lediglich der mit dem Mandanten geschlossene Vertretungsvertrag (ist) privatrechtlich, im übrigen ist der Rechtsanwalt völlig in die staatliche Rechtspflege eingebaut. *

Der hochaktuelle Reformvorschlag stammt vom NS-Rechtswahrerbund aus dem 
Jahr 1937. Diesem Rechtserneuerer-Bund gehörte seit 1933 Rechtswahrer Scharpenseel an, der (im Beschwerdesenat des BGH neben Cartellbruder Meyer) noch 1975 rechtserneuert hat: Ausschließen von Verteidigern und Hauptverhandlung ohne Angeklagte.

\section{Zur Beweisaufnahme}

Die Beweisaufnahme ist das Kernstück des Strafprozesses. Das Stammheimer Gericht hat das Beweiserhebungsverfahren insbesondere zum Beweisvereitelungsverfahren fortentwickelt.

I. Beweisvereitelungsmethode:

Nach bisherigem Recht, wie es noch der 1977 erschienene Großkommentar zu $\ 199$ StPO begreift, galt: "Die vollständige Vorlage der Ermittlungsakten bildet eine unentbehrliche Voraussetzung für die dem Vorsitzenden und dem Gericht zugewiesene Tätigkeit. « (Dementsprechend hat der Verteidiger unbeschränkte Akteneinsicht, $\$$ I47 StPO.)

Statt dessen Stammheim 1975-1977: sorgfältige Selektion von Ermittlungsakten, wobei die Strafverfolgungsbehörden nur knapp die Hälfte der vorhandenen als verfahrenserheblich würdigen und vorlegen, worauf das Gericht die wiederholten Verteidigeranträge, den aktenkundigen Strafverfolgern »die vollständige Vorlage* aufzugeben, mit der Einsicht zurückwies, daß die Bundesanwaltschaft schon wissen werde, was sie zu tun und zu lassen habe.

2. Beweisvereitelungsmethode:

Die Bundesanwaltschaft legt streng geheime Akten an, welche die Verteidigung nicht kennenlernen darf, weil sonst das Wohl der ganzen Republik gefährdet werden würde. Wird aber durch Zufall die Existenz einer Geheimakte entdeckt, dann läßt die Bundesanwaltschaft den Bundesminister der Justiz die Akten vor den Verteidigern sperren, damit nicht diese durch Aktenkenntnis das Staatswohl schädigen. Außerdem dient das auch der Verfahrensbeschleunigung, die von sehr vielen Verfahrensbeteiligten dringend gewünscht wird: z. B. von Regierungsmitgliedern und allen anderen Politikern, von Kolumnisten und vielen Leserbriefschreibern.

3. Beweisvereitelungsmethode:

Kommt die Verteidigung drauf, daß ein Amtsträger zu diesem Verfahren mehr weiß als die Bundesanwaltschaft möchte, daß die Verteidigung es weiß: dann bewirkt sogenannte Amtshilfe, daß der Amtsträger als Zeuge ein Aussageverbot mitbringt, wonach er auf die zweite Frage schon nicht mehr antworten darf. Aber dagegen haben die Verteidiger den Rechtsweg, mindestens zwei Instanzen, und können dann an der Nordsee und am Rhein und im Münsterland Verbotsbefreiungsprozesse führen.

4. Beweisvereitelungsmethode:

Das Gericht sagt dem Verteidiger, er soll sich seine Zeugen selber laden, was der Verteidiger tut, und wenn der Zeuge da ist, schickt ihn das Gericht wieder fort, weil es ihn nur sehen aber nicht hören wollte.

5. und wichrigste Beweisvereitelungsmethode:

Weil es in der Bundesrepublik keine politischen Strafprozesse gibt, sondern nur gemeine Verbrecher abgeurteilt werden (obgleich das aus Stammheim jeder weiß, 
hat Bundesjustizminister Vogel es vorgestern »bekräftigt", SZ vom 25. 4. 1977),

kommt es auf die Würdigung einer sbestimmten Gesinnung، oder spolitischer Vorstellungen gar nicht an;

weil 'gemeine Verbrecher keine Gesinnung haben, sondern bloß Mordlust oder Explosionswollust (nach Bundesanwalt Widera), kommt es auf politische Entwicklungen und Zielvorstellungen überhaupt nicht an;

und ,wir haben den liberalsten Staat, den es jemals im Gebiet der Bundesrepublik gab und wahrscheinlich einen der liberalsten in der Welt< (nach Bundesanwalt Zeis) - deswegen ist in Stammheim für Gesinnungen kein Raum;

und der Indochina-Krieg der USA hat mit Stammheim nichts zu tun - und wer weiß denn am Ende dieser Verhandlung wirklich noch, ob er überhaupt stattgefunden hat?!

An diesem Punkt des Verfahrens spätestens hat das Gericht offengelegt, daß es, wenn es ernsthaft die von der Anklage behauptete Täterschaft in Betracht zog:

Sachaufklärung und Wahrheitsermittlung nicht im Sinne hatte;

statt dessen allenfalls mechanistische Rekonstruktion;

und, bestenfalls, die Angeklagten auf Objektstatus zu reduzieren.

Ich erinnere an die Anträge der Verteidigung vom 4. Mai r 976 und an die Präsentation der hierzu sachverständigen Zeugen Osborne, Agee, Thomas, Peck durch die Verteidigung am 22. 6. 1976; dazu lapidar der Senat: „daß die benannten Beweisthemen unter keinem rechtlichen Gesichtspunkt, auch nicht zur Begründung eines Rechtfertigungsgrundes, von Belang sind. Der Vietnam-Krieg ist nicht Gegenstand dieses Verfahrens. * (TN IOI52)

VIII. Das Vorurteil als Endurteil

Daß das Stammheimer Gericht vieljähriger öffentlicher Vorverurteilung gerecht werden wird, hat keiner von uns zu irgendeiner Zeit bezweifelt; um so weniger, als stets Experten sich finden, die einen und wieder einen Anschlag auf unseren freiheitlichen Rechtsstaat exakt bis in die Stammheimer Zellen zurückverfolgen können.

Aufgabe der Verteidigung war notwendig erstrangig Schutz für das Leben der Gefangenen.

Die Schlußvorträge der Ankläger vom 5. bis 7. Oktober 1976 sind über ihr erklärtes Ziel, die Höchststrafe, weit hinausgeschossen: in ihnen hat sich Vernichtungswille artikuliert; der Eifer, moralisch zu vernichten, die Angeklagten zu entmenschlichen, sie zu verteufeln, $\mathrm{Haß}$, Abscheu, Verachtung auf sie zu lenken. Eineinhalb Jahre einer demonstrativ parteilichen Verhandlungsleitung durch Richter Prinzing hatten die Sitzungsvertreter der ihrem Selbstverständnis nach sobjektivsten Behörde der Welt enthemmt zu Exzessen, wie ich sie in einem deutschen Gerichtssaal nach Freisler nicht für möglich gehalten hätte, wie andererseits ich sie vor- und wiedergefunden habe in dem materialreichen Buch des Bundesanwalts in Ruh Dr. Walter Wagner mit dem Titel: „Der Volksgerichtshof $\propto$.

Da reicht dem Oberstaatsanwalt Holland nicht der Superlativ: "schwerstkriminelle Gewaltverbrecher vom Schlage der Gudrun Ensslin - da beförderte er »auf die tiefste Stufe sittlicher Wertung - findet, noch tiefer, eine Gesinnung, in der nichts Menschliches mehr ist - erblickt da "seelische Abgründe, die auch langjährige Strafrechtspraktiker erschaudern lassen* - gewiß jedenfalls den Zuhörer solcher Tiraden.

Als sein Amtsbruder Widera mit seinen Anklagen unstillbarer Mordlust - »mög- 
lichst viele Menschen töten", "möglichst viele amerikanische Soldaten (sollten) getötet werden * - *auf niedrigster Stufe*, lustmörderisch *Detonationen . . . einem Orgasmus ähnlich mit sinnlicher Freude erlebt « - seine Klimax erreicht hatte, kam er auf seine Füße zurück und klagte wegen des Verzehrs mexquisiter Lebensmittel in großen Mengen « an sowie des Tragens einer "Lederjacke aus besonders feinem Leder" wegen - um dahinter zu entdecken: den "Teufelskreis, gedacht, Terror durchzusetzen«, um »für die Bevölkerung als Ganzes die Freiheit (zu) beseitigen .

Da holte Ankläger Zeis gleich weiter aus: für Baader außerhalb der Anklageschrift noch einen Mord extra, nämlich an Holger Meins; und kurzerhand per Schlußvortrag Erweiterung der Anklage nach $\int_{129}$ StGB auf sämtliche in diesem Verfahren tätig gewesenen und noch tätigen Rechtsanwälte; denn zu dem Anklagepunkt ,Fortführung der kriminellen Vereinigung aus der Haft anwaltliche Sündenregister.

Daneben saß sichtlich zufrieden der Prinzing-Senat und hörte sich serienweise Beleidigungen von Prozeßbeteiligten an: Angeklagten und ihren gewählten Verteidigern. Der Catcher-Stil der Riege Zeis, Widera, Holland schien ihm in diese Hauptverhandlung durchaus zu passen.

Wer in jenen Tagen das zynische Trumphieren zügelloser Redefreiheit und die brutale Hetze dieser Ankläger erlebt hat: mochte begreifen, daß da offene Feindschaft regierte. Wer Zeis im Geifer seines Gefechts erlitten hat, der den seit Ulrike Meinhofs Tod der Hauptverhandlung ferngebliebenen Baader beiläufig noch der Tötung des Holger Meins bezichtigte - $\bowtie$ Baader hat den Tod des Holger Meins auf dem Gewissen, wenn er ein solches hat « -, konnte in diesem Ausbruch dessen Ursprung erkennen und danach fragen: auf wessen Betreiben denn eigentlich war die längst richterlich angeordnete Verlegung des Holger Meins von Wittlich in die Krankenstation von Stammheim unterblieben? Baaders oder Zeis'?

Und wo Ankläger Zeis "klar « ist, daß Ulrike Meinhofs Tod "Selbstmord war «, fällt mir die Frage ein: wer eigentlich hatte drei Jahre vor ihrem Tod Röntgenaufnahmen ihres Schädels, Szintigraphie ihres Gehirns, erforderlichenfalls Zwangsnarkose in Auftrag gegeben (12.6.1973, I BJs 6/71): Frau Meinhof oder vielleicht der Oberstaatsanwalt Zeis? Wer hat, damit der Auftrag ausgeführt werden könne, der Wahrheit zuwider beschwichtigt: die Eingriffe seien nicht gesundheitsgefährdend und könnten auch im Gefängnis vorgenommen werden (29.6. 1973, I BJs 6/7I)? - Frau Meinhof oder Herr Zeis?

Wer eigentlich hat, nachdem Ende Juli/Anfang August 1973 eine Fülle von Warnungen öffentlich geworden war: die in Auftrag gegebenen Eingriffe gefährdeten Gesundheit und Leben der Patientin, auf den Fortgang jener "Untersuchung " gedrängt (9. 8. 1973, I BJs 6/73): Frau Meinhof oder Herr Zeis?

An diesem *Selbstmord $*$ ist schon vieles nklar $*$ : nur nicht, daß es einer war. Sollte das Herr Zeis nicht wissen?

Längst hat Stammheim den Anschein verloren, Justiz finde hier statt:

die Rechtsprechung ist an Gesetz und Recht gebunden,

die Richter sind unabhängig und nur dem Gesetz unterworfen,

Ausnahmegerichte sind unzulässig,

festgehaltene Personen dürfen weder seelisch noch körperlich mißhandelt werden, die Todesstrafe ist abgeschafft.

Als am 7. 4. Richter Berroth in voller Kenntnis der wiederholten ärztlichen Forderungen, unverzüglich die Haftbedingungen von der Isolierung weg zu lockern, um die Gefangenen lebensfähig zu erhalten, die Totalisolation verfügt und trotz Durst- 
streiks als Gegenwehr aufrecht erhalten hat: hat er die Gefangenen seelisch und körperlich mißhandelt.

Am 1 4. 3. 1977 hat der Stammheimer Anstaltsarzt dem Gericht geschrieben:

wNachdem seit den letzten Stellungnahmen der Herren Professoren Ehrhardt vom 29. 10. 1975, Mende vom 29. 10. 1975, Müller vom 3. I1. 1975 und Rasch vom 7. 11.1975 fast achtzehn Monate verstrichen sind und die Fortdauer der Untersuchungshaft unter nahezu gleichbleibenden Bedingungen zweifellos keine Besserung des psychischen Befindens zur Folge hat, werden die Empfehlungen der genannten Ärzte nachdrücklich wiederholt. ... Infolgedessen ist es meines Erachtens dringend erforderlich, die derzeitigen Haftbedingungen zu korrigieren, und zwar in Ubereinstimmung mit den Ausführungen des Herrn Prof. Dr. med. Mende vom 29. I0. I975 . . des Herrn Prof. Dr. med. Müller vom 3. I I. I 975 . . , sowie des Herm Prof. Dr. med. Rasch vom 17. 11 . 1975 , dessen Ausführungen zufolge rdie entscheidenden Behandlungsmaßnahmen ... in einer Änderung der Haftbedingungen mit Ermöglichung größerer sozialer Interaktionen` liegen; sschwer vorstellbar dürfte . . . sein, die bei den Angeklagten bestehenden Isolationsfolgen therapeutisch anzugehen, ohne die jetzigen Haftbedingungen grundlegend zu ändern` und daß ,die Durchführung einer Behandlung . . . bei Beibehaltung der jetzt gegebenen Haftbedingungen nicht möglich sein dürftec,«

Am 29. 3. 1977 sind die Gefangenen in unbefristeten Hungerstreik getreten mit der Mindestforderung: daß sie nach den Forderungen aller von den Gerichten in den Prozessen gegen die RAF bestellten Gutachter zu interaktionsfähigen Gruppen von mindestens is Gefangenen zusammengefaßt werden.

Am 30. 3. 1977 hat der Stammheimer Anstaltsarzt an das Gericht geschrieben:

"Sofern im Verlauf des Hungerstreiks eine hierdurch bedingte Lebensgefahr eintreten sollte, sind zwangsweise durchgeführte Behandlungsmaßnahmen kaum noch erfolgversprechend, sondern es ist vielmehr mit dem tödlichen Ausgang des Hungerstreiks zu rechnen.

Nach den derzeitigen Erkenntnissen ist eine durch den Hungerstreik bedingte Lebensgefahr mit letztlich letalem Ausgang nur dadurch zu verhindern, daß die bereits in meinem Schreiben vom 14 . 3. 1977 für erforderlich gehaltenen Veränderungen der Haftbedingungen unverzüglich in Angriff genommen und durchgeführt werden.*

Am 30. 3. I977 hält der Anstaltsleiter, Herr Nusser, in einem Gesprächsvermerk fest:

*Auf ausdrückliche Frage erklärte Baader hierbei, der Hungerstreik habe das Ziel, die seir langem aufgestellten Forderungen nach Veränderungen der Haftbedingungen, die von ailen Gutachtern unterstützt würden, durchzusetzen.*

Am 4. 4. 1977 gibt der Stammheimer Anstaltsarzt einen Vermerk über Telefongespräch mit Verteidiger Temming an das Gericht:

-Ich habe darauf erwidert, daß infolge des Hungerstreiks jederzeit der Zustand einer akuten Lebensgefahr eintreten kann.*

In seinem fachärztlichen Gutachten vom 6. 4. 1977 hat Prof. Rasch dem Vorstand der JVA Stammheim unter anderem geschrieben:

»Objektiv - das heißt aus medizinischer Sicht - ist bei den drei Untersuchten gegenüber dem Befund vom August 1975 eine weitere Verschlechterung des Gesundheitszustands eingetreten.

Nach dem überschaubaren Verlauf ist davon auszugehen, daß die festzustellende Verschlechterung des Gesundheitszustandes der Untersuchten unmittelbare Folge der besonderen Haftbedingungen ist, denen sie ausgesetzt sind. Um Wiederholungen zu vermeiden, verweise ich auf meine oben zitierten Stellungnahmen (vom 10. 9., 22.9. und 7. I I. 1975), auf den Inhalt der am 23. 10. 1975 beim 2. Strafsenat des Oberlandesgericht Stuttgar durchgeführten Besprechung sowie auf meine Veröffentlichung, Die Gestaltung der Haftbedingungen für politisch motivierte Täter in der Bundesrepublik Deutschland، (Monatsschrift für Kriminologie, Sommer 1976 )...

Bei Beibehaltung der jetzigen Haftbedingungen, die nicht das notwendige Maß sozialer Interaktionen gewährleisten, ist bei den Untersuchten ein weiteres Fortschreiten der gesundheitlichen Schäden zu erwarten, das schließlich zur Haftunfähigkeit führt.*

Am I 5. 4. 1977 hat der Stammheimer Gerichtsvorsitzende verfügt: 
•Die Anträge ..., die Haftbedingungen der Angeklagten anders zu gestalten, werden abgelehnt. « Aus den Gründen: „Von den Angeklagten ist eine erhebliche Gefährdung für die Sicherheit und Ordnung in der Anstalt und darüber hinaus zu befürchten. So verbieter sich zunächst der unbeschränkte Umgang der Angeklagten mit den anderen Anstaltsinsassen. Es kommt also von vonrherein nur eine abgesonderte Unterbringung der Angeklagten und eine Einschränkung des Verkehrs mit anderen Anstaltsinsassen in Betracht.

Ferner: die Verlängerung des Umschlusses von $16 \mathrm{Uhr}$ auf 22 Uhr ist organisatorisch nicht möglich; Lichtverlängerung über 23 Uhr hinaus auch nicht.

Auch eine zeitliche Ausdehnung der Möglichkeit zu Verteidigerbesuchen ist nicht erforderlich (!). Die eingehende Kontrolle der Verteidiger vor Besuchen bei den Angeklagten ist unumgänglich. Das Gericht bedauert das, kann aber über die Gefahren, die ein unkontrollierter oder weniger kontrollierter Verkehr für die Sicherheit und Ordnung in der Anstalt mit sich brächte, nicht hinwegsehen.

Abschließend sei bemerkt, daß keine Rede davon sein kann, es gehe von den bestehenden Haftbedingungen Lebensgefahr aus." (Es folgt die Unwahrheit, das Gericht habe den Angeklagten - entsprechend den Empfehlungen der Arzte! - die Möglichkeit zu erweiterten zwischenmenschlichen Kontakten seit Jahren eingeräumt).

Kennt Richter Foth nicht die Gutachten der Professoren Rasch, Mende, Müller, Schröder aus den Monaten September bis November 1975?

Hatte Richter Foth am I5. 4. vergessen, was der Anstaltsarzt am 14.3. ihm geschrieben hatte?

Hat Richter Foth den Brief vom 30. 3. des Anstaltsarztes an ihn nicht zur Kenntnis genommen oder kennt er ihn und nimmt seit vier Wochen nunmehr den Tod der Gefangenen in Kauf?

Woher weiß der Richter Foth, die bestehenden Haftbedingungen könnten nicht eines Tages lebensgefährlich werden? Erscheint ihm nicht ratsam, in dieser Frage lieber dem Prof. Rasch als Fachmann zu folgen, der bei Beibehaltung der jetzigen Haftbedingungen mit Haftunfähigkeit rechnet?

Bis zum Beweis des Gegenteils müssen wir davon ausgehen, daß Haftrichter Foth weiß, was er tut:

Mit seiner haftrichterlichen Verfügung vom I s. 4. 1977 gegen die einhelligen Forderungen der Arrte hat er Andreas Baader, Gudrun Ensslin und Jan Carl Raspe zum Tod verurteilt.

\section{Heldmann}

\section{AUS DEN ANLAGEN*:}

1. Aus Anlage I (Einstellungsantrag vom 7. 2. 1977 wegen Mitwirkung des ungesetzlichen Richters):

(I) Die Blätrer I 3 I 56 und I 3 I 57 der Tonbandniederschrift bew eisen, daß Bundesrichter Mayer gegenüber den Angeklagten in diesem Verfahren und den von ihnen gewählten Verteidigern befangen war (sein Schreiben vom 20. 7. 1976 an Dr. Kremp); auch und gerade als Beschwerderichter am 3. Strafsenat des BGH. Als stellvertretender Senatsvorsitzender und Berichterstatter war Bundesrichter Mayer maßgeblich beteiligt an dem Beschluß vom 22. 10. 1975 , nach $\$ 23$ Ia StPO die Hauptverhandlung in diesem Verfahren ohne die Angeklagten fortzusetzen.

In jenem Beschluß finden sich u. a. die folgenden Ausführungen:

*Bedingt durch zahlreiche - auch die angebliche Befangenheit der Mitglieder des Gerichts betreffende - Verfahrensanträge und schwere Störungen der Hauptverhandlung seitens der Angeklagten hat die Beweisaufnahme bisher nicht begonnen.* (Blatt 2)

\footnotetext{
- Die Anlagen konnten aus Platzgründen nur insoweit abgedruckt werden, wie zum Verständnis der Nachrede uneriäßlich ist. (D. Red.)
} 
* Seine Auslegung ( $\$ 23$ Ia StPO) darf nicht dazu führen, daß die Absicht des Gesetzgebers vereitelt wird, dem Tatrichter die Durchführung der Hauptverhandlung auch gegen solche Beschuldigte zu ermöglichen, die den staatlichen Organen jede Achtung versagen und mit allen Mitteln den geordneten Ablauf des Verfahrens zu stören suchen. « (Blatt 4)

"Sie (die Gutachten der psychiatrischen Sachverständigen) ergeben auf alle Fälle, daß die Hauptursachen des Zustandes der Angeklagten in deren eigenem Verantwortungsbereich liegen." (Blatt I2) (Anmerkung hierzu: Jeder, der diese Gutachten kennt, weiß, daß diese Behauptung eine völlige Umkehrung der Tatsachen ist.)

"Die Beschwerdeführer leben unter anderen Haftbedingungen. Sie müssen Beschränkungen auf sich nehmen, die nach dem Urteil von Prof. Rasch durch die ihnen gewährten Privilegien nicht aufgewogen werden. Indes haben sie diese ihre Verhandlungsunfähigkeit mitbedingenden Umständen selbst zu verantworten. Die Beschwerdeführer gehören einer zahlenmäßig verschwindend geringen Gruppe der Bevölkerung an, die es im Gegensatz zu dieser für unerläßlich hält, den gewiß in mancherlei Hinsicht verbesserungsbedürftigen Zustand der Gesellschaft in der Bundesrepublik Deutschland - wie übrigens jeder Gesellschaft - nicht mit dem demokratischen Mittel der Uberzeugung der Wähler, sondern gegen deren Willen unter Anwendung rücksichtsloser Waffengewalt zu verändern. Ihr augenscheinlich durch nichts zu beeinflussendes realitätsfernes Bild von den gesellschaftlichen Verhältnissen und von den tatsächlichen Möglichkeiten, auf sie einzuwirken, verführt sie zu einer fanatischen Verfolgung ihrer Ziele auch aus der Untersuchungshaft heraus. ...

Aus dieser Haltung heraus haben sie in der Haft nicht nur mit Hilfe durch Rechtsanwälte verbreiteter Zellenzirkulare zum Zwecke der Aufrechterhaltung des Zusammenhalts ihrer Vereinigung den Kontakt zu inhaftierten Gesinnungsgenossen aufrechterhalten, sondern es auch verstanden, Kampfanweisungen an in Freiheit befindliche Terroristen gelangen zu lassen." (Blatt I 3)

"Die Gefährlichkeit der Beschwerdeführer, die in den genannten Umständen zum Ausdruck kommt, ließ den für die Gestaltung der Untersuchungshaft verantwortlichen Stellen keine andere Wahl ais die, dem durch eine entsprechende Verschärfung der Haftbedingungen Rechnung zu tragen. ...

Wenn sie gleichwohl seit Jahren das Verhalten fortsetzen, das die staatlichen Organe zur Anwendung dieser Haftbedingungen zwingt, so haben sie somit die Herbeiführung ihrer Verhandlungsunfähigkeit in Kauf genommen. Das genügt zur Annahme vorsätzlichen Verhaltens im Sinne des $\$ 23$ ia Abs. I StPO.« (Blatt I )

"Die Beschwerdeführer müssen sich wie jeder Rechtsunterworfene mit unanfechtbaren Entscheidungen abfinden. Daß sie es nicht tun, liegt an ihrer grundsätzlichen Nichtachtung rechtsstaatlicher Entscheidungsprozesse und deren unter rechtstreuen Bürgern friedenstiftenden Funktion und ist ihnen daher zuzurechnen. Die Argumentation der Verteidigung, die diesen Zusammenhang leugnet, läuft auf die Zumutung hinaus, den Angeklagten entweder durch entsprechende Haftbedingungen die Fortsetzung ihrer kriminellen Vereinigung einschließlich der Vorbereitung ihrer Befreiung zu erleichtern oder auf die Durchführung einer Hauptverhandlung gegen sie zu verzichten. Das kann nicht rechtens sein.\& (Blatt I6)

Diese Textstellen sind als Bestandteile einer richterlichen Zwischenentscheidung noch vor Beginn der Beweisaufnahme in der Hauptverhandlung eine einzigartige Dokumentation richterlicher Voreingenommenheit.

Der BGH-Beschluß vom 22. xo. 1975 zu $\$ 23$ ra StPO heißt, zusammengefaßt, in seinem Kern: Wegen der besonderen Gefähriichkeit dieser Angeklagten, insbesondere wegen der Fortführung krimineller Vereinigung aus der Haft heraus mit Hilfe von Verteidigern, haben sie Haftbedingungen zu erleiden, welche für ihre Verhandlungsunfähigkeit zumindest mit-ursächlich sind.

$\mathrm{Daß}$ diese Haftbedingungen vom jeweils ersten Tag der Haft an bereits exekutiert worden waren, verschweigt jener Beschluß, führt damit irre über das wahre Verhältnis von Ursache und Wirkung.

Voreingenommenheit gegen die Angeklagten und ihre uneingeschränkte Vorverurteilung durch die beteiligten Richter ist dem Beschluß vom 22. 10. 1975 der Bundesrichter Scharpenseel, Mayer, Schauenburg unmittelbar abzulesen.

Vor Kenntnis der Beschlußgründe war den Angeklagten in diesem Verfahren die am 10. 1 . 1977 offenkundig gewordene Befangenheit des Bundesrichters Mayer nicht mit einer für Richterablehnung hinreichenden Bestimmtheit bewußt.

Der Brief vom 20.7.1976 vom Bundesrichter Mayer an WELT-Chefredakteur Kremp nunmehr belegt dessen Befangenheit gegenüber den Angeklagten in diesem Verfahren und Verteidigern ihrer Wahl:

-In derselben Sache wende ich mich heute wiederum an Dich. Vorige Woche ist in Stgt.- 
Stammheim das frühere Bandenmitglied Gerhard Müller als Zeuge vernommen worden. Ich übersende Dir als Anlagen

I. auszugsweise Ablichtungen der kriminalpolizeilichen Vernehmung Müllers (S. 46, 95, I 80 ), 2. Auszug aus dem (vom Tonband übertragenen) Wortprotokoll vom 13 . Juli 76.

Der ,kleine Dicker ist der in Entebbe getötete Wilfried Böse. Daß es sich bei dem von ihm übergebenen Papier um den wenige Tage später der Meinhof abgenommenen Ensslin-Kassiber handelte, hat sich in der Verhandlung klar ergeben.

Möchte sich die , Welt ^ nicht unter dem Aspekt dieser neuen Erkenntnisse noch einmal mit dem Aufsatz im 'Spiegel، vom 4.9.72 (Nr. 37) S. 67 befassen?

Nicht um meinetwillen, sondern um einmal wieder die Haltung und die Praktiken dieses Blattes deutlich werden zu lassen, das sich seinerzeit mit eilfertiger Bereitwilligkeit die - wie sich nun zeigt - von Ströbele und Müller ausgeheckte Entlastungslegende zu eigen machte und das den Baader-Meinhof-Leuten soviel publizistische, gelegentlich sogar materielle Unterstützung (Honorare für Interviews aus der Untersuchungshaft) zuteil werden ließ. Vielleicht könnte diese Aufgabe gar einen Chefredakteur reizen?

Zum - etwa noch nötigen - besseren Verständnis der Zusammenhänge füge ich eine Abschrift des damaligen Beschlusses des Bundesgerichtshofs bei. Der handschriftliche Vermerk auf dem Wortprotokollauszug stammt übrigens vom Vorsitzenden und bezieht sich eben auf Schily. [...] Ergebnis:

Der Beschluß des BGH zur Fortsetzung der Hauptverhandlung in Abwesenheit der Angeklagten ist ungesetzlich zustande gekommen, weil Bundesrichter Mayer seiner Pflicht zur Selbstablehnung nicht nachgekommen ist $(§ 30 \mathrm{StPO})$. [ . . ]

2. Aus Anlage 2 (Einstellungsantrag vom 9. 4. 1977 wegen Abhören von Verteidigergesprächen):

Mit seinem Schreiben vom 18. 3. 1977 an den Senat hat Justizminister Bender das Abhören von Verteidigergesprächen während 22 Tagen zugegeben. [...]

In der Hauptverhandlung am 22. 3. hat der Vorsitzende Richter erklärt: "Prozessual freilich ist der geschehene Verstoß gegen $\ 148$ StPO nicht aus der Welt zu schaffen.\& (Bl. 13722 der Tonbandniederschrift)

Richtig ist, daß dieser Verfahrensverstoß irreparabel ist.

Richtig ist darüber hinaus, daß er für diese Hauptverhandlung fortwirkt.

Wichtig ist ferner, daß die Gefahr jederzeitiger Wiederholung offen und mehrfach angezeigt worden ist (I. 3., I. 9., I. IO., I. Ir.).

Als unstreitig ist anzunehmen, daß der Hinweis des zuständigen Haftrichters an die Justizverwaltung auf die Fortgeltung des $\ \mathrm{I}_{4} 8 \mathrm{StPO}$ auch in diesem Verfahren nicht mehr als deklamatorischen Charakter hat. Die Abhör-Minister haben nie geleugnet, $\$$ I 48 StPO schon im März i 975 als geltendes Recht gekannt zu haben.

Sowohl das vergangene Abhören von Verteidigergesprächen als auch die seine Wiederholung unverblümt anzeigende Programmvorschau begründet Verfahrenshindernis gegenüber der Fortührung dieser Hauptverhandlung. [...]

3. Aus Anlage 6 (Einstellungsantrag - Schily - vom 5. 6.1975 wegen Entzug des gesetzlichen Richters):

Die Bestellung von Dr. Prinzing zum Vorsitzenden für das vorliegende Verfahren kam auf folgende Weise zustande:

Auf Weisung der Bundesregierung in Ubereinkunft mit der Landesregierung in Baden-Württemberg wurde im Jahre 1973 Stuttgart als Prozeßort von der Bundesanwaltschaft bestimmt.

Vorsitzender des 2. Strafsenats des Oberlandesgerichts Stuttgart, der für das vorliegende Verfahren nach dem Geschäfesverteilungsplan zuständig ist, war im Jahre 1973 der Vorsitzende Richter Hänle. Dieser erschien der Bundesregierung, dem Generalbundesanwalt und der Landesregierung von Baden-Württembergg nicht geeignet, in dem Verfahren gegen die Rote Armee Fraktion tätig zu werden. Aus diesem Grunde wurde zwischen dem ehemaligen Generalbundesanwalt Martin und dem Justizminister von Baden-Württemberg, Traugott Bender, im Jahre 1973 nach Absprache mit der Bundesregierung vereinbart, den Vorsitzenden 
des 2. Strafsenats des Oberlandesgerichts Stuttgart auszuwechseln und anstelle des bisherigen Vorsitzenden Hänle den Vorsitzenden Richter am Landgericht Dr. Prinzing zu berufen, weil dieser über "Erfahrungen in Monstre-Prozessen, Durchblick, Durchsetzungsvermögen und erkennbaren Ehrgeiz * verfüge. Um den Wechsel im Vorsitz des 2 . Strafsenats herbeizuführen, mußte zunächst eine entsprechende Richterstelle für den bisherigen Vorsitzenden Hänle freigemacht werden. Dementsprechend wurde dem bisherigen Vorsitzenden des 1. Strafsenats des Oberlandesgerichts Stuttgart, Xaver Hoch, nahegelegt, sich um den Posten eines Ministerialdirigenten im Justizministerium von Baden-Württemberg zu bewerben. Er wurde in die besondere Situation eingeweiht, die durch die Wahl von Stuttgart als Prozeßort entstanden war. Der Vorsitzende Richter des I. Strafsenats, Xaver Hoch, erklärte sich daraufhin bereit, den Vorsitz des I. Strafsenats aufzugeben und sich, obwohl er bisher eine solche Bewerbung nicht in Erwägung gezogen hatte, für die Stelle im Justizministerium zu bewerben. Nachdem eine Unterredung über den bezeichneten Themenkreis zwischen Justizminister Bender, dem damaligen Generalbundesanwalt Martin, dem Generalstaatsanwalt beim OLG Stuttgart Weinmann und dem ehemaligen Oberlandesgerichtspräsidenten Henn stattgefunden hatte, reichte der Vorsitzende Richter beim OLG Sturtgart Xaver Hoch seine Bewerbung ein. Der Bewerbung wurde innerhalb relativ kurzer Frist entsprochen. Mit der Ernennung des bisherigen Richters Hoch zum Ministerialdirigenten wurde die Stelle des Vorsitzenden des I. Strafsenats frei, für die sich nunmehr entsprechend den getroffenen Absprachen der bisherige Vorsitzende des 2. Strafsenats, der Zeuge Hänle, bewarb. Auch dieser Bewerbung wurde entsprochen und Hänle wurde zum Vorsitzenden Richter des I. Strafsenats des OLG Stuttgart ernannt. Auf diese Weise war der Vorsitz im 2. Strafsenat vakant geworden. Gemäß den internen Vereinbarungen zwischen dem Generalbundesanwalt und dem Justizminister des Landes Baden-Württemberg bewarb sich für den Vorsitz Dr. Theodor Prinzing, der bisherige Vorsitzende Richter einer Jugendstrafkammer. Er wurde, wie vorgesehen, am 4. 2. 1974 zum Vorsitzenden Richter des 2. Strafsenats ernannt und damit zum Vorsitzenden in dieser Hauptverhandlung berufen.

4. Aus Anlage 7 (Beschwerdeschrift vom 6. 10. 1975 wegen fehlender Verhandlungsfähigkeit der Angeklagten)

Der Senat verwirft [...], ohne sich mit ihnen auseinander gesetzt zu haben, die Gutachten seiner eigenen Sachverständigen, wo diese zu den Ursachen für die zeitliche Begrenzung der Verhandlungsfähigkeit Stellung nehmen (S. s):

Das internistische Gutachren schließt im Ergebnis die Hungerstreiks als ursächlich gerade aus (S. IO-I 2); das Gutachten Mende zieht sie als ursächlich nicht einmal in Betracht (S. 8 f.); das Gutachten Rasch zieht sie zwar in Betracht, um jedoch sogleich, kontrapunktisch, die "für den modernen Vollzug völlig ungewöhnlichen Haftbedingungen* als ursächlich zu kennzeichnen (S. $6 \mathrm{f}$.$) .$

Der Senat hat seine eigene "Sachkenntnis*, welche ihn drei Monate lang die Angeklagten als voll verhandlungsfähig erkennen ließ, an die Stelle derjenigen der von ihm selbst bestellten Sachverständigen gesetzt.

Er verletzt damit einmal mehr seine Wahrheitsermittlungspflicht und seine prozessuale Fürsorgepflicht.

\section{ANMERKUNG}

Die Angeklagten im Stuttgart-Stammheimer Prozeß haben sich selbst zu den Bombenanschlägen auf amerikanische Dienststellen in der Bundesrepublik bekannt und die Verantwortung für die Sprengung im Springer-Hochhaus übernommen. Die Aktionen sind Straftaten, die nach dem StGB geahndet werden. Sie bleiben es, auch wenn man sie - wie Otto Schily es in seinem Plädoyer getan hat - in den Zusammenhang des Vietnam-Krieges stellt. Die Aktionen der RAF und der ihrem Beispiel folgenden Gruppen sind der Eigengesetzlichkeit solcher fatalen Aktionen erlegen. Der Schaden, der durch diese Aktionen im politischen Klima der Bundesrepublik angerichtet wird, ist unabsehbar. Unschuldige mußten ihr Leben lassen. 
Politisch aber wurde in erster Linie die Linke in der Bundesrepublik getroffen und geschwächt.

Die Angeklagten sind selbst ein Opfer dieses veränderten politischen Klimas geworden. Stuttgart-Stammheim setzte in der Bundesrepulbik ein Zeichen. Angesichts der gefährlichen, politisch motivierten Straftaten wurden grundlegende prozessuale Sicherungen herausgedreht: in den Vordergrund trat die unmittelbare Bekämpfung eines vorab verurteilten Feindes.

Hiergegen formuliert Hans Heinz Heldmann sein Plädoyer. Er besteht als engagierter Liberaler auf der Unschuldsvermutung, auf der Waffengleichheit zwischen Anklage und Verteidigung, auf der Trennung von Strafsanktion und moralischer Verurteilung, auf dem Anspruch, daß ein Befangener nicht richten darf. Angesichts der Realität des Verfahrens und des Unvermögens, die Preisgabe rechtsstaatlicher Sicherungen rückgängig zu machen, bleibt für Heldmann nichts als der Protest. Dazu gehört, daß Heldmann - nicht zuletzt wegen der illegalen Uberwachung von Gesprächen der Angeklagten mit ihren Verteidigern, die der Innenminister von Baden-Württemberg, Karl Schieß, win vergleichbaren Situationen wiederum in gleicher Weise « durchzuführen versprach - nicht mehr im Gerichtssaal, sondern in einem Hotel plädierte: Es gibt keinen Adressaten, der folgenreich reagieren könnte. So wendet sich Heldmann an die Restbestände liberaler Offentlichkeit in diesem Lande. Heldmann will aufrütteln. Er versucht das mit der schon abgegriffenen Münze des Vergleichs mit dem deutschen Faschismus; er verzichtet in diesem Schlußvortrag nicht auf verbale Uberschärfungen.

Angesichts der rechtsstaatswidrigen Verfahrensverstöße redet Heldmann im Zorn, in Verzweiflung. Es ist auch unser Zorn. Doch wer nicht unmittelbar dem "Stammheimer-Landrecht" ins Auge sehen mußte, der weiß, daß der Zorn die Stimme verschlägt. Der muß fragen: Vermindern derartige Formen von overstatement (die nur diejenigen erreichen, die schon wüberzeugt " sind) nicht die noch gegebenen Einflußchancen? Sie können zum Bumerang werden. Muß man nicht, wenn man Gegenöffentlichkeit gegen ein weiteres Umkippen des Strafprozesses mobilisieren will, den Zorn kühlen und nüchtern, vielleicht sogar spröde an Detailfragen das festmachen, was gegenwärtig in den Grauzonen der Bundesrepublik passiert?

Joachim Perels / Jürgen Seifert 